\title{
Tactile feedback enhanced hand gesture interaction at large, high-resolution displays
}

\author{
Stephanie Foehrenbach *, Werner A. König, Jens Gerken, Harald Reiterer \\ University of Konstanz, $\mathrm{HCl}$ Group, Box D-73, 78457 Konstanz, Germany
}

A R T I C L E I N F O

Keywords:

Hand gestures

Tactilc feedback

Fitts' Law

Controlled experiment

Imput device

Large high-resolution display

\begin{abstract}
A B S T R A C T
Human beings perceive their surroundings based on sensory information from diverse chamnels. However, for human-computer interaction we mostly restrict the user on visual perception. In this paper, we contribute to the investigation of tactile feedback as an additional perception modality. Therefore, we will first discuss existing user studies and provide a classification scheme for tactile feedback techniques. We will then present and discuss a comparative evaluation study based on the ISO 9241-9 [Ergonomic requirements for office work with visual display terminals (VDTs) - Part 9: requirements for non-keyboard input devices, 2000]. The 20 participants performed horizontal and vertical one-directional tapping tasks with hand gesture input with and without tactile feedback in front of a large, high-resolution display. In contrast to previous research, we cannot confirm a benefit of tactile feedback on user performance. Our results show no significant effect in terms of throughput (effective index of performance (IPe)) and even a significant higher error rate for horizontal target alignment when using tactile feedback. Based on these results, we suggest that tactile feedback can interfere with other senses in a negative way, resulting in the observed higher error rate for horizontal targets. Therefore, more systematic research is needed to clarify the influencing factors on the usefulness of tactile feedback. Besides these results, we found a significant difference in favor of the horizontal target alignment compared with the vertical one in terms of the effective index of performance (IPe), confirming the work by Dennerlein et al. [Force feedback improves performance for steering and combined stcering-targeting tasks, in: $\mathrm{CHI}$ '00: Proceedings of the SIGCHI Conference on Human Factors in Computing Systems, ACM, New York, NY, USA, 2000, pp. 423-429].
\end{abstract}

\section{Motivation}

In application domains where collaboration, presentation, or the exploration and analysis of large information spaces are predominant tasks, large high-resolution displays are widely used. These wall-sized displays offer great opportunities for information visualization [22] and improve user orientation and search performance [3], but

\footnotetext{
* Corresponding author. Tel.: +497531883704; fax: +497531884772.

E-mail address: Stephanie.Foehrenbach@uni-konstanz.de (S. Foehrenbach).
}

also require more physical navigation [2]. Since the display characteristics match or even exceed the capabilities of the human visual system in terms of resolution or field of view [13], users have to move in front of these displays to gain either in-depth knowledge or an overview. Therefore, input devices and interaction techniques are needed which allow a more flexible interaction from any point and distance.

Inspired from previous research such as $[5,6,21]$, we investigated hand gesture input as an interaction technique which meets this mobility requirement but also offers a very natural and direct way of interaction. We believe that hand gestures are particular well suited 
for interaction with large displays, as with those displays users have the possibility to step away from the display in order to perceive overview information. Such distant user positions substantiate the need for input modalities which allow distant interaction, a requirement which can be addressed with hand gesture input. Smaller displays do not require the user to step back as much, hence the need for distant interaction is reduced and direct touch is probably a more appealing input modality. Based on the linguistic findings of Kendon [11] and previous evaluation studies of Vogel and Balakrishnan [21], we identified pointing and selection gestures and implemented a tracking library for gesture recognition in combination with a commercial finger tracking device (see Section 3).

However, a general problem for the interaction with large high-resolution displays still remains. Since the display capabilities may exceed the human visual acuity, users cannot solely rely on visual feedback. Imagine a user moving backwards to a distant position to get an overview of the displayed information space: from there it is hard to visually perceive and therefore almost impossible to select a standard-sized button or menu item. In the following paper, we therefore will address the question if tactile feedback in addition to visual feedback can complement visual information. Thus, we investigated the effect of tactile feedback on user performance with a controlled experiment based on the ISO standard 9241-9. The 20 participants performed horizontal and vertical onedirectional tapping tasks with hand gesture input with and without tactile feedback for target crossing. In Section 4, we describe the experimental design in detail while we present and discuss the results in Sections 5 and 6 . In the following Section 2, we discuss related work and findings on tactile feedback for pointing devices and provide a classification scheme for tactile feedback techniques.

\section{Tactile feedback for pointing devices}

When reviewing related work on tactile and forcefeedback-enhanced interfaces, several terms are used interchangeably [9]. For the purpose of this work, we define tactile feedback and distinguish it from force feedback based on the ISO 9241-9 [10], the human somatosensoric system and following the distinction found in [9]. The international ISO standard 9241-9 [10] holds ergonomic requirements and recommendations for the design of non-keyboard input devices. In addition to guiding the design of input devices, the standard describes in its Annex B several procedures for assessing the efficiency and effectiveness of input devices (e.g. facilitating a one- or multi-directional tapping task to assess pointing performance). According to the ISO 9241-9 [10]. tactile feedback is described as the "indication of the results of a user action transmitted through the sense of touch". We refine this definition and incorporate the qualities of the sense of touch, according to the foundations in the human somatosensoric system, and extend it to also take into account the impact on user movement.
Thereby, tactile feedback can be classified and distinguished from force feedback:

- Tactile feedback describes sensations which are applied to the skin and perceived by the human sense of touch including vibration, pressure, stretching, and touch [16]. Tactile feedback, unlike force feedback cannot restrict user movements.

- Force feedback applies force with various strengths to the user and can actively restrict users in their movements if the applied force is large enough. The feedback is sensed through the sense of touch and proprioception.

Scheibe et al. [18] observed that enhancing hand gesture interaction with tactile feedback seems to increase the reliability of interaction tasks. In a pilot study, eight participants were asked to perform common interactions in a virtual car cockpit using the corresponding real-world gestures while wearing a tactile data-glove system. Tactile feedback, sensed as an ongoing vibration on the fingertips, was given when contact of a virtual object with a finger occurred. Tasks were performed with and without the additional feedback. Results showed that participants clearly preferred the tactile system and it was observed that particular small, almost by the real hand occluded objects were operated with greater reliability when tactile feedback was given. Hence, tactile feedback seems to improve hand gesture interaction; however, the outcome of the study neither gives evidence of the detailed impact on performance nor on error rate nor on movement time.

Other areas in the field of human-computer interaction already make use of tactile feedback. Braille displays allow visually impaired users to explore the internet, mobile phones vibrate when a text message is received, and input devices give tactile clues like the discrimination between keys on keyboards. By comparing the results of a typing task performed by typists and casual users using a conventional and a piezo electric flat keyboard Barret and Krueger [4] found out that the performance of both user groups was significantly higher with the conventional keyboard. The used piezo electric mechanism within the flat keyboard detects when pressure is applied to a key area by the user, which causes a change in electric potential and constitutes an actuation signal. Here, lack of the familiar haptic feedback (kinesthetic feedback through key travel and tactile through key discrimination) directly decreases the performance. Effects of enhancing keyboard interaction with a stylus on a PDA with tactile feedback were evaluated by Brewster et al. [7]. Participants performed a text entry task once in a laboratory and once in an underground train. A vibrotactile actuator at the back of the device was used to generate two different stimuli which were used to either indicate a successful button press or signal an error. Results showed that tactile feedback improved the number of corrected errors significantly in both settings, reduced the error rate in the laboratory setting and lead to a lower overall workload of the participants who preferred the tactile system over the non-tactile system. Another evaluation considering 
pen-based input was conducted by Forlines and Balakrishnan [9]. Here, tactile feedback was added directly to the stylus. In a selection task, they did not only study the effect of different feedback conditions (tactile plus visual vs. visual only) but also direct vs. indirect input and selecting using pointing vs. crossing. In the direct input condition, the stylus input and the output display surface are coincident, whereas in the indirect input condition they are separated. With selection through pointing, the user selects a target in tapping into its area, whereas with crossing the user selects a target with crossing through the target boundary. Tactile feedback was given to confirm a successful selection. The authors discovered that although tactile feedback did not show significant beneficial effects for all conditions, it improved the selection time for indirect pointing and direct crossing selection tasks. This outcome suggests that tactile feedback, while having the potential, does not per se guarantee for an improved interaction but that the accompanied interaction technique also influences the benefits of tactile feedback. Akamatsu and MacKenzie [1] found that the performance of a modified mouse could be improved through additional tactile feedback. In the tactile feedback condition, a solenoid-driven pin stimulated the tip of the index finger once the cursor overlapped the target area. The feedback was turned off when the target was selected, or the cursor was moved outside of the target area. Note that this differs from the feedback in Forlines and Balakrishnan [9] as it is given before the user performs a selection task. Compared with the other feedback conditions, results showed that tactile feedback lead to the highest index of performance with $6.4 \mathrm{bits} / \mathrm{s}$.

Based on these mixed results, it seems to be critical to distinguish between different forms of tactile feedback when discussing its usefulness. We identified two different approaches on how to provide tactile feedback:

1. Proactive feedback: the feedback is given prior to a certain interaction and indicates a call for action by the user. This means that as soon as a tactile feedback is sensed, the user has to perform a (predefined) action (e.g. click on an object). It might be that the tactile feedback is given until the action is performed. The cited studies by Akamatsu and MacKenzie [1], Scheibe et al. [18], and Barret and Krueger [4] can be classified in this category.

2. Retroactive feedback: in this case, tactile feedback is given after an interaction has been performed by the user. Here, we have to distinguish between two different kinds of feedback. Positive feedback means that tactile feedback is given to indicate that an interaction or task was performed correctly. Negative feedback means that tactile feedback is given to indicate an error or mistake, requiring the user to repeat or correct the action. The cited study by Forlines and Balakrishnan [9] belongs to the positive retroactive feedback category while the study of Brewster et al. [7] provides both, positive and negative feedback.

Summarizing the results of the different studies, the proactive feedback seems to increase performance or at least user satisfaction while the results for the retroactive feedback are more mixed. The study by Brewster et al. [7] might suggest that negative retroactive feedback has a higher influence on user performance. However, more research in this area is needed to clarify this issue.

However, in case of combining tactile feedback with hand gesture interaction for WIMP or similar interfaces, the proactive feedback approach seems to be more promising. In such a case, the user needs help in pointing to and selection of an object. Since the computer does not know which object the user is interested in, giving retroactive feedback is not possible. Hence, we combined the hand gesture interaction, which will be described in detail in the following section, with proactive tactile feedback in a similar way as Akamatsu and MacKenzie [1].

In our evaluation scenario, it is known which target the user aims at. However, this is not generally the case. Hence, the question raises how to provide proactive feedback in cases where the intended target is not known in advance. Predicting which target the user aims at in order to ease target selection is a research question on its own and different methods have been proposed by researchers. Dynamically expanding targets analyze the distance between cursor position and target area and increase in size if the cursor is moved towards them, respectively, decrease if the distance is enlarged (see [15] for an evaluation on the effects of expanding targets on performance in selection tasks). Such expanding targets are strongly connected to the underlying application due to the need of semantical knowledge on target locations. For cases where such semantically knowledge is not available and the distance between cursor position and target area cannot be analyzed, methods which analyze cursor movement characteristics (e.g. the velocity) have been proposed to predict if users are aiming at a target [14]. We think that similar mechanisms, either based on cursor to target distance, cursor movement velocity or a combination of both could be applied for triggering tactile feedback if the aimed target is not known in advance.

\section{Hand gestures}

Kendon [11] describes a variety of every day gestures which are used in combination with speech. These kinds of gestures are interesting for human-computer interaction, as they are already known by the potential users and could therefore lead to a decreased learning effort and a better recall when used for interaction.

In the context of human-computer interaction, a pointing operation positions the cursor on a display with the user facing it; hence, gestures that are used in this manner should be used. Kendon identified the use of the extended index finger and open hand where " $[\ldots]$ pointing gestures are regarded as indicating an object, a location, or a direction, which is discovered by projecting a straight line from the furthest point of the body part that has been extended outward, into the space that extends beyond the speaker." [11]. Even if both gestures share the same semantic theme the usage is slightly different. An extended index finger is used when one specific object 


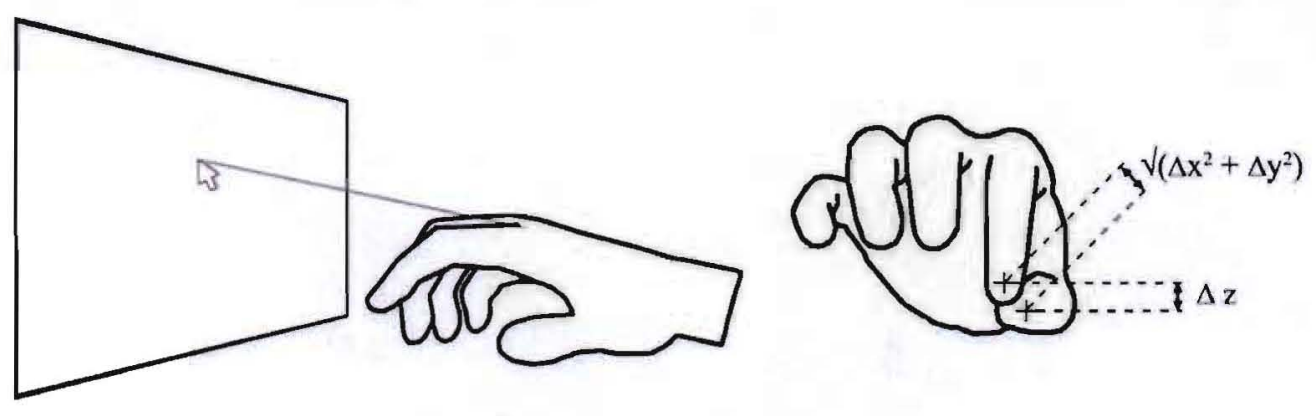

Fig. 1. Pointing gesture (left) and selecting gesture (right).

or location is referred to, whereas pointing with the open hand indicates that the object is related to the topic but is not explicitly mentioned. The exact location of a specific object is what users aim for when positioning the cursor over a target, which describes the usage of the extended index finger in pointing. Even if the semantic meaning would be identical, this gesture bears some drawbacks when used for interaction. Vogel and Balakrishnan [21] evaluated three combinations of point and click hand gestures and found that pointing with the extended index finger showed the highest error rate and the lowest ease of use score ( 1 out of 12). Another drawback is that this gesture requires higher tension than pointing with the open hand, which makes the open hand gesture a better candidate considering biomechanical load in this comparison. These drawbacks discourage the usage of the extended index finger gesture for pointing. Also, the usage would resemble every day gesticulation, not as much as the extended index finger, but considering the discussed issues of both gestures, the open hand seems to be the best choice for being used as a pointing gesture. We therefore used the open hand with an absolute mapping for cursor positioning, where a straight line, defined by the orientation of the palm, was projected and intercepted with the display. The cursor was placed at the interception (see Fig. 1). This is in line with Kendon's view on pointing gestures described above. Furthermore absolute positioning, compared with relative, prevents users from losing track of the cursor, which is reported as a usability problem on large displays by Robertson et al. [17].

To interact with objects, a selection gesture is needed as well. Ideally, such a gesture should fit well when used in combination with the pointing gesture and should furthermore be already well known from every day gesticulation or similar "selection" actions. Besides pointing gestures, Kendon [11] also describes the so-called R-Family of precision grip gestures that are used when the speaker wants to be very exact and precise about something and therefore special attention is needed. Selecting in the context of human-computer interaction shares this meaning, as the user wants to exactly select one specific object of the application. When performing a gesture of the R-Family the tips of the index finger and the thumb are brought together to form a shape that resembles a circle or a ring, a finger movement that can be used in combination with our selected pointing gesture quite well. Yet another advantage is that the movement of the gesture
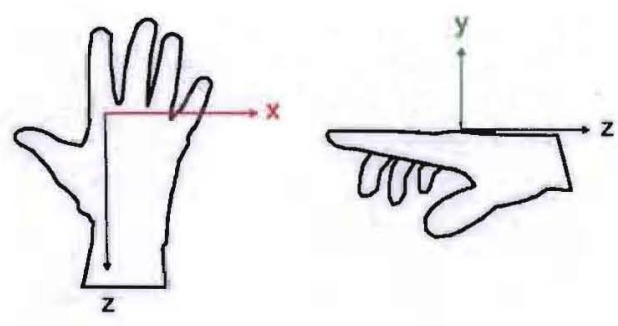

Fig. 2. Local hand coordinate system used for gesture recognition.

mimics the action of doing a left mouse click with every computer user being familiar with. It also provides implicit feedback due to the fingertip contact signaling that the gesture has been performed. We picked this gesture to be used for performing a selection because of the similar meaning, the additional relation to simple mouse click actions, and the implicit feedback. A selection, which is mapped to a single left mouse click, is triggered when the distance of the index finger and thumb tip fall below predefined thresholds of two metrics (see Fig. 1) based on the $3 \mathrm{D}$ position of the fingertips located in a "local hand coordinate system" (see Fig. 2). The 3D positions of the fingertips are derived from the output data of the used tracking solution, described in Section 4.1.

\section{Experiment}

We conducted a controlled experiment to assess and compare the usability of the presented hand gestures with and without tactile feedback as an input device for large high-resolution displays. This section describes our experimental settings and hypothesis.

\subsection{Materials}

The experiment took place in front of the Powerwall of the University of Konstanz, a large high-resolution display. The Powerwall of the University of Konstanz is a wallsized display with a resolution of $4640 \times 1920$ pixels and a physical dimension of $5.20 \mathrm{~m} \times 2.15 \mathrm{~m}$. It uses a multi projector system with soft-edge blending and it is equipped with an optical tracking system developed by A.R.T. This tracking system uses six infrared cameras to cover the area in front of the display. The cameras are able 
to identify the position and movement of markers that can be placed on persons, e.g. to assess their current location and use this as an input variable. In combination with a non-intrusive data-glove, also developed by A.R.T, this system was used for finger tracking. The data-glove was enhanced with several markers on the back of the hand as well as on three fingers - the latter were attached similar to foxgloves (see Fig. 3). This construction enabled the tracking of the exact position of one's hand as well as single fingers. If every marker is visible for the cameras, this system reaches an accuracy of $<1 \mathrm{~mm}$. We used this commercial data-glove solution, as it can be accustomed to most of the hand and finger sizes and should therefore be adjustable to fit most participants. Furthermore, hygienic issues, arising from the use by many different users, are minimized, as unlike other data-glove solutions (e.g. the CyberGlove" II, 5DT Data Glove 14 UItra) only a small area of the hand and finger is in contact with the data-glove solution. We modified the attachment of the marker on the back of the hand to improve the visibility of the marker for the cameras and therefore increase the tracking quality. In order to provide a tactile feedback, we used an extension of this system described in [18]. Around the inside of the three fingertips covered by the markers, the so-called shape memory alloy wires were attached. The used shape memory alloy wires have the ability to alter their shape due to changes in their temperature; they shorten if heated and return to their original length when cooling down. The implemented feedback mechanism uses this shape altering characteristic to create the impression of a continuous vibration. A wireless connection provided the possibility to attach a low voltage to the wires in order to heat them. The tasks (see Section 4.2) were presented and interaction was recorded via IEval, a

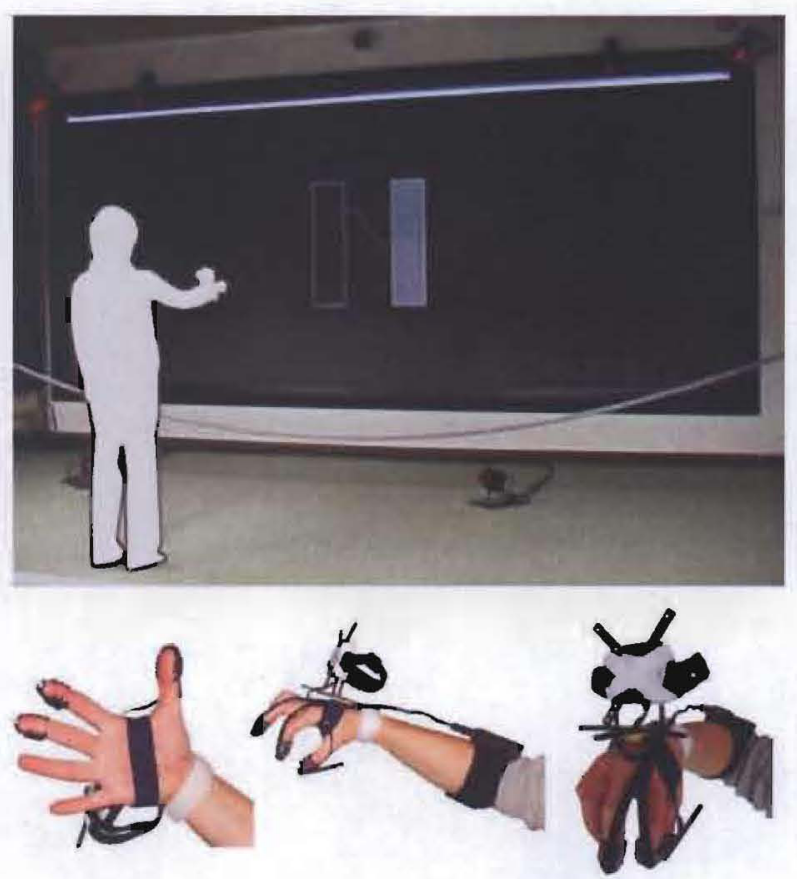

Fig. 3. Powerwall (top) and data-glove (down) software tool that can be used for pointing device experiments [12]. To accommodate for the natural hand tremor, we integrated a band-pass filter that provides dynamic smoothing of the interaction without restricting fast movements. This decreases the effect of the interaction itself on the accuracy. We designed a short pre-test questionnaire to assess the participants' prior experience as well as some demographic data. For subjective assessment of the different experimental conditions, we used the questionnaire provided by the ISO $9241-9$ [10], which asks participants to rate one device and then rate the second device in comparison to the first device. Users rated the non-tactile as the first device (absolute measurement) and the tactile feedback as the second device (relative to the non-tactile variant). The questionnaire consists of items like overall satisfaction as well as accuracy and fatigue of fingers/wrist/arm, etc. In total, it comprises 12 items that have to be rated on a 7-point scale.

\subsection{Tasks}

We based our experiment on Fitts' Tapping Task as described and suggested by ISO $9241-9[10]$ to assess the performance of pointing devices. These tests are widely used and accepted (see [20] for a review). We used the one-directional tapping task that consists of two rectangular targets that are furthermore varied in terms of their width $(W)$ and the amplitude $(A)$ between them. This onedirectional tapping task enabled us to evaluate the effect of vertical vs. horizontal movement direction. Participants were asked to click on each of these targets in an alternating manner as fast and precise as possible. This "clicking" was done by using the selection gesture illustrated in Section 3. In the tactile condition, tactile feedback was provided while the cursor overlapped the target area. Since we wanted to evaluate whether tactile feedback can improve pointing performance compared with visual feedback alone (non-tactile feedback condition), the goal was to minimize the latency between the user action (moving the cursor over the target) and the tactile feedback. Thereby, we initiated the tactile feedback without any latency. However, there is a technical limitation which introduces a latency: it takes about $50 \mathrm{~ms}$ (maximum) until the wires reach the necessary temperature to initiate the vibration. The tactile feedback to the user's tips of the active fingers (index and thumb) was turned off only after selecting the target or after the cursor was moved outside of the target area. This integration of the tactile feedback is based on the work by Akamatsu and MacKenzie [1] who provided tactile feedback in a similar way while testing an enhanced mouse. In the non-tactile condition visual feedback only, provided by the icon of the cursor, was available for the participants to judge whether the cursor overlapped the target area or not. We furthermore varied the target alignment, using horizontal as well as vertical aligned targets (see Fig. 4).

To cover a wide set of difficulties that can be encountered when interacting in front of the Powerwall, 
we initially used $3(W) \times 3(A)$ combinations for horizontal tasks and $2(W) \times 2(A)$ combinations for vertical tasks. The latter was due to the limited vertical size of the Powerwall ( $2.15 \mathrm{~m}$ compared with the $5.20 \mathrm{~m}$ in horizontal) and the necessity that participants may also "overshoot" a target. Larger amplitudes or target widths for vertical tasks may have otherwise resulted in participants performing a selection gesture outside of the display. The exact pixel-values can be seen in Fig. 5 as well as the resulting indexes of difficulty. However, during the experiment, we observed that participants moved themselves to a larger extent in front of the display than expected, triggering the tracking cameras ineffective for the outer parts of the display. Therefore, we had to exclude this amplitude for further analysis, resulting in a $3(W) \times 2(A)$ combination for horizontal tasks and the corresponding reduction in terms of the index of difficulty from 5.6 bits maximum to 4.6 bits maximum (see Fig. 5 ).

\subsection{Hypothesis}

This section describes our experimental hypothesis as well as their foundation in the current literature.

\subsubsection{H1: tactile vs. non-tactile}

We assumed that tactile feedback would result in a significant performance improvement, expressed by the effective index of performance (IPe) measurement. This

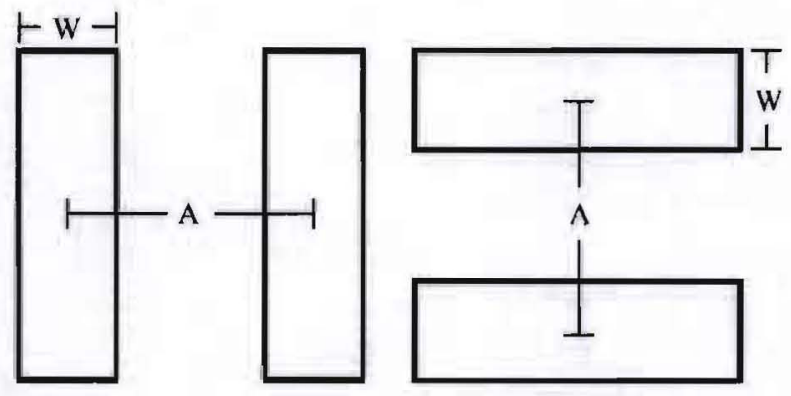

Fig. 4. Horizontal (left) and vertical (right) alignment of tapping targets. hypothesis is in line with the literature review presented in Section 2 that strongly suggests that proactive tactile feedback is able to improve user performance in many ways, ranging from lower error rates to lower movement times. The effective index of performance includes both, movement time and error rates (see [20] for details) and therefore provides an appropriate measurement for this hypothesis.

\subsubsection{H2: horizontal vs. vertical target alignment}

We assumed that the index of performance for horizontal targets (see Fig. 4, left) would be significantly higher compared with the vertical target alignment (see Fig. 4, right). This hypothesis is in line with findings by Dennerlein et al. [8]. In an experiment featuring a tunnel steering task, conducted via a stationary mouse and a regular display, they observed that users were able to guide the mouse cursor more quickly through horizontal areas of the task compared with the vertical areas. They ascribed this effect to differences in the joint kinematics, in particular to the multi-joint coordination. In a similar way, horizontal and vertical hand movement also relies on different muscles and joints, therefore we expected similar results. In the Dennerlein study, they were able to neutralize the effect via a force-feedback mechanism that guided users to stay in the tunnel. We were therefore curious if and how tactile feedback might have an effect in our experiment as well on this phenomenon.

\subsection{Experimental design}

We used a $2 \times 2$ within subjects design with feedback (tactile, non-tactile) and target alignment (horizontal, vertical) being the independent variables. A latin square design was used for counter-balancing in order to address possible effects of sequence, learning or fatigue. Our participants were randomly assigned to one of the resulting four experimental groups. As dependent variable we used the measurements provided by ISO 9241-9 [10], namely movement time (MT, in ms), error rate (ERR in \%), a

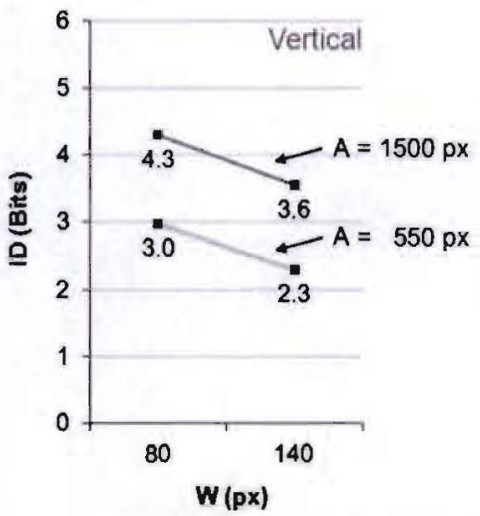

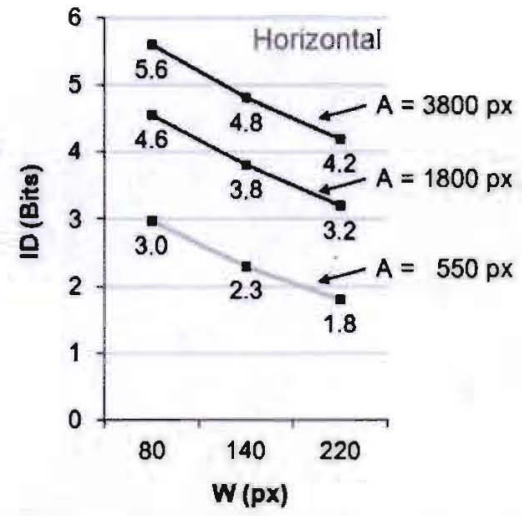

b

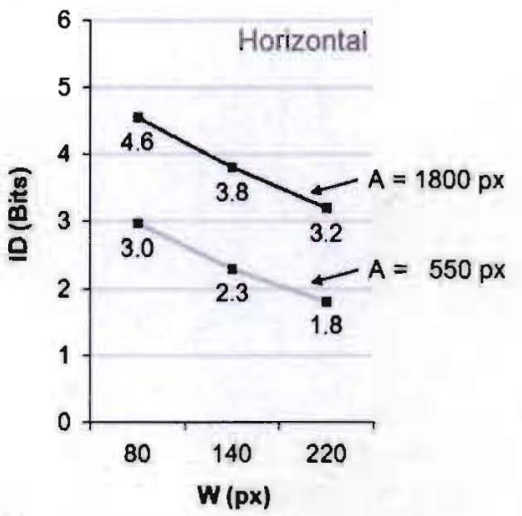

Fig. 5. (a) Initial design of $W \times A$ combinations and resulting index of difficulties (different shades: amplitudes, $x$-axis: target sizes, left: horizontal, right: vertical). (b) Resulting $W \times A$ combinations for horizontal alignment after exclusion of one amplitude condition ( 3800 pixels). 
and the effective index of performance (IPe in bits/s). The latter combines the movement time and error rate in one single measurement. Although we instructed participants to be as fast and precise as possible, they still may use different strategies, either focusing on precision or speed. The effective index of performance provides the necessary measure to take this aspect into account. Unlike movement time covering only the speed of the interaction or error rate covering only the precision, the effective index of performance covers the efficiency as well as the effectiveness of pointing performance. The measures were calculated using the following formulas:

Index of difficulty: ID $=\log ^{2}(A / W+1)$

Effective width : We $=S D(\Delta 0) \times 4.133$

Effective index of difficulty : IDe $=\log ^{2}(A / W e+1)$

Effective index of performance : $\mathrm{IPe}=\mathrm{IDe} / \mathrm{MT}$

As can be seen in Eq. (4), the effective index of performance is based on movement time (MT) and an adjusted index of difficulty, called effective index of difficulty (IDe). This effective index of difficulty (see Eq. (3)) takes into account the observed accuracy in using the effective target width (We), which is derived from the movement endpoint standard deviations (see Eq. (2)).

\subsection{Participants}

We selected 20 participants to take part in our experiment. Of those, 15 were male and five were female. The average age was 30.8 years with a standard deviation of 9.9 years. All of them were regular computer users, while 13 already had some experience with large displays (standard projector or the Powerwall). None of the participants had prior experience with a data-glove or something similar.

\subsection{Procedure}

Each session started with the pre-test questionnaire. Users were then equipped with the data-glove followed by a short functionality test of the tactile feedback. In the next step, participants were asked to step in the center in front of the Powerwall, $3 \mathrm{~m}$ away from the display. They were instructed about the interaction, the gestures they should use to interact, and to be as fast and precise as possible.

A training session was started then to facilitate learning and allow participants to become accustomed to the interaction techniques. A test monitor gave support during the training session if necessary. This training session consisted of a full block of vertical and horizontal tasks as well as non-tactile and tactile feedback, whereas the sequence was based on the participants assigned test condition. During training we used $2(W) \times 2(A)$ combinations and ten trials for each combination, resulting in 160 trials. The selection of the reduced $W \times A$ combinations was done based on the goal to keep the training rather short and at the same time to reach similar difficulty levels as in the following real tasks. During these each participant completed two blocks of the assigned condition, and now 16 trials for each $W \times A$ combination, resulting in 832 trials. All participants together completed 16,640 trials of which 12,820 trials were used for analysis, due to the tracking problem mentioned in Section 4.2.

After completion of the tapping test, participants were asked to fill in the ISO 9241-9 [10] questionnaire. The experiment lasted in total about $1 \mathrm{~h} /$ session and participants were given 5 EUR as compensation.

\section{Results}

This section describes the analysis and results of our experiment. We started our analysis by calculating the model fit, averaged across all participants, for Fitts' Law. Results show that we have a very high model fit for each of the factor combinations, with $r^{2}$ constantly above 0.99 . Therefore, we can assume that the Fitts' Law model fits quite well for our experiment.

Our first hypothesis stated a significant difference in favor of the tactile feedback in terms of the effective index of performance (IPe). Results of our RM-ANOVA however show that this is not the case. For both horizontal and vertical target alignment, the non-tactile feedback performed better, however the differences are very small and not significant (horizontal means: 3 bits/s non-tactile vs. $2.99 \mathrm{bits} / \mathrm{s}$ tactile, SD: $0.29 \mathrm{bits} / \mathrm{s}$ vs. $0.31 \mathrm{bits} / \mathrm{s}$; vertical means: 2.53 bits $/ \mathrm{s}$ non-tactile compared with $2.46 \mathrm{bits} / \mathrm{s}$ tactile, SD: 0.23 bits/s vs. 0.28 bits/s, see Fig. 6 ). Therefore, we have to reject our hypothesis in favor of the null hypothesis, stating there is no significant difference.

Our second hypothesis stated a significant difference in favor of the horizontal target alignment compared with

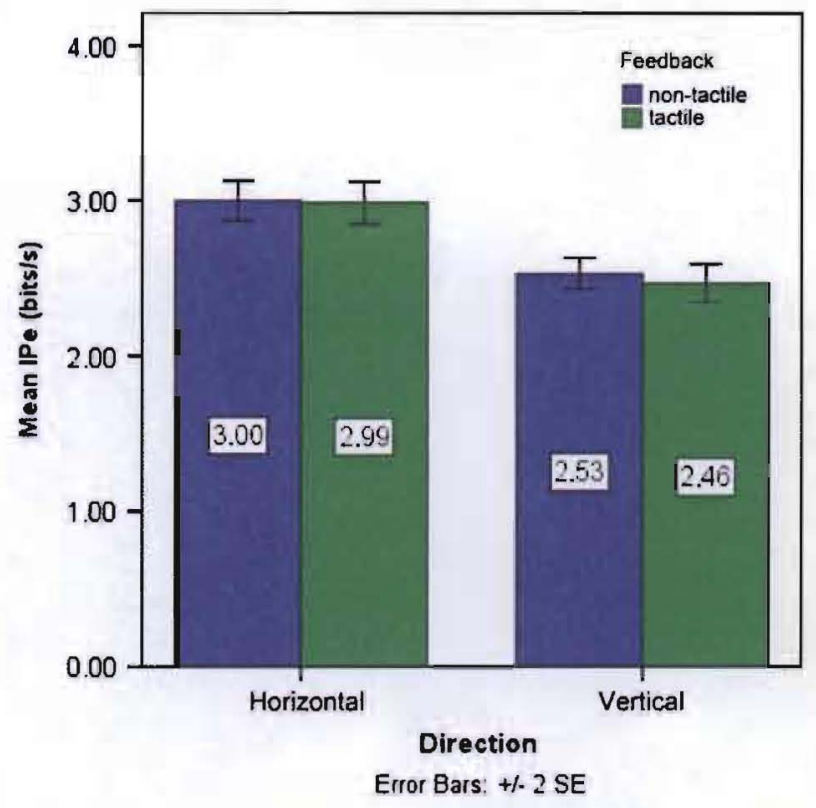

Fig. 6. Effective index of performance for horizontal and vertical target alignment. 
the vertical one in terms of the effective index of performance. As it turns out, this is indeed the case $\left(F_{1,19}=124.857 p<0.001\right.$, horizontal mean: $2.99 \mathrm{bits} / \mathrm{s}, \mathrm{SD}$ : $0.29 \mathrm{bits} / \mathrm{s}$ vs. vertical mean: $2.49 \mathrm{bits} / \mathrm{s}, \mathrm{SD}: 0.25 \mathrm{bits} / \mathrm{s}$ ). Therefore, we can accept our hypothesis. However, in contrast to the study by Dennerlein et al. [8], the tactile feedback did not compensate for these differences. We further analyzed the effect of the tactile feedback in terms of error rate and movement time. Results show that the movement time is slightly lower for both vertical and horizontal target alignment when providing the user with tactile feedback. However, these differences are not significant (see Fig. 7).

Regarding the error rate results look different (see Fig. 8). For the horizontal target alignment, we discovered a significant higher error rate when using tactile feedback $\left(F_{1,19}=9.17, p=.007,10 \%\right.$ vs. $12 \%$, SD: $4.8 \%$ vs. $6.2 \%$ ) - for vertical alignment the difference was not significant $\left(F_{1,19}=2.61, p=.112\right)$.

Regarding the subjective feedback derived from the questionnaire our participants rated nearly the entire items positive for the non-tactile feedback (with the

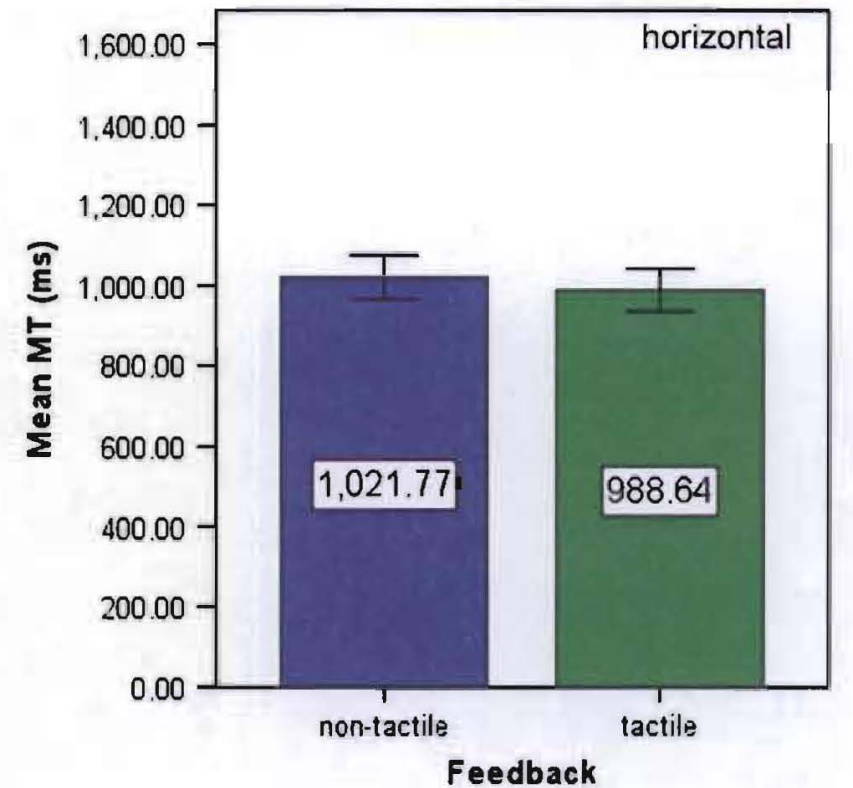

Error Bars: $+1-2$ SE

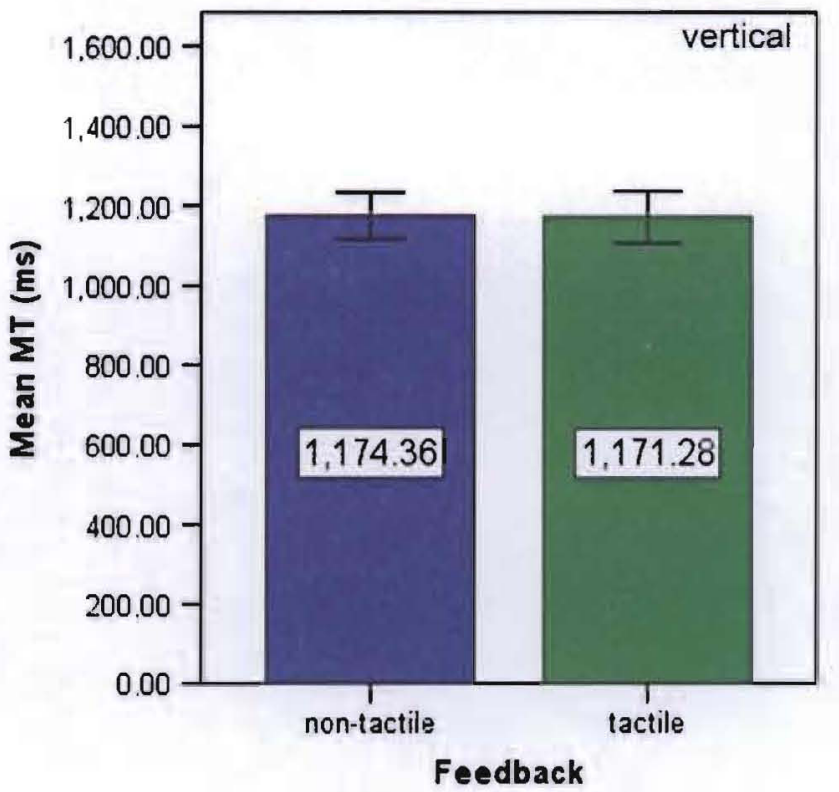

Error Bars: +/-2 SE

Fig. 7. Influence of tactile feedback on movement time.

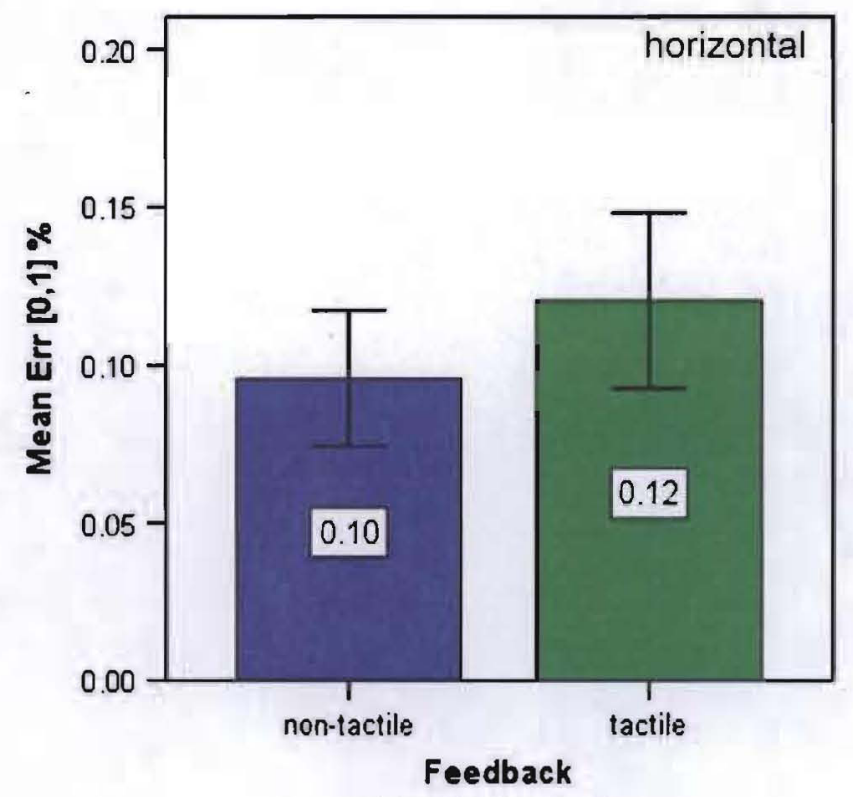

Error Bars: $+/-2$ SE

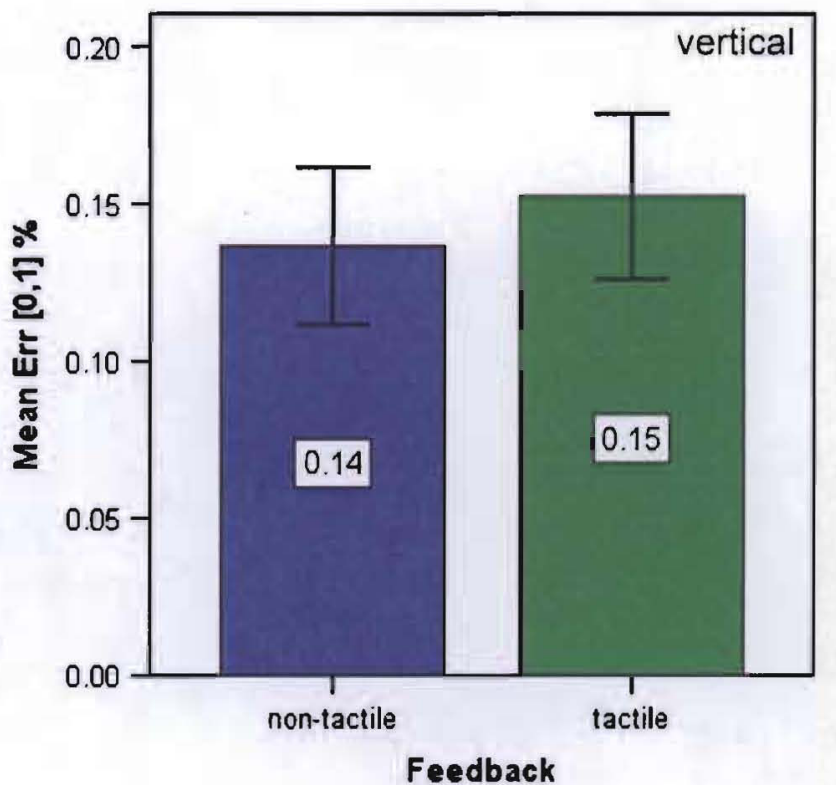

Error Bars: +/-2 SE

Fig. 8. Influence of tactile feedback on error rate. 
exception of arm fatigue, see Fig. 9). The second part of the questionnaire asks to rate the tactile feedback relative to the non-tactile. Results show that our participants either liked or disliked the tactile feedback, resulting in three nearly discrete groups (7 dislikes, 7 likes, 6 undecided, see Fig. 10). We looked for correlations between task performance and whether a participant was in the "I like tactile" or "I dislike tactile" group. However, there was no significant effect.

\section{Discussion}

While the findings from $[1,18]$ discussed in Section 2 suggest that proactive tactile feedback may improve user

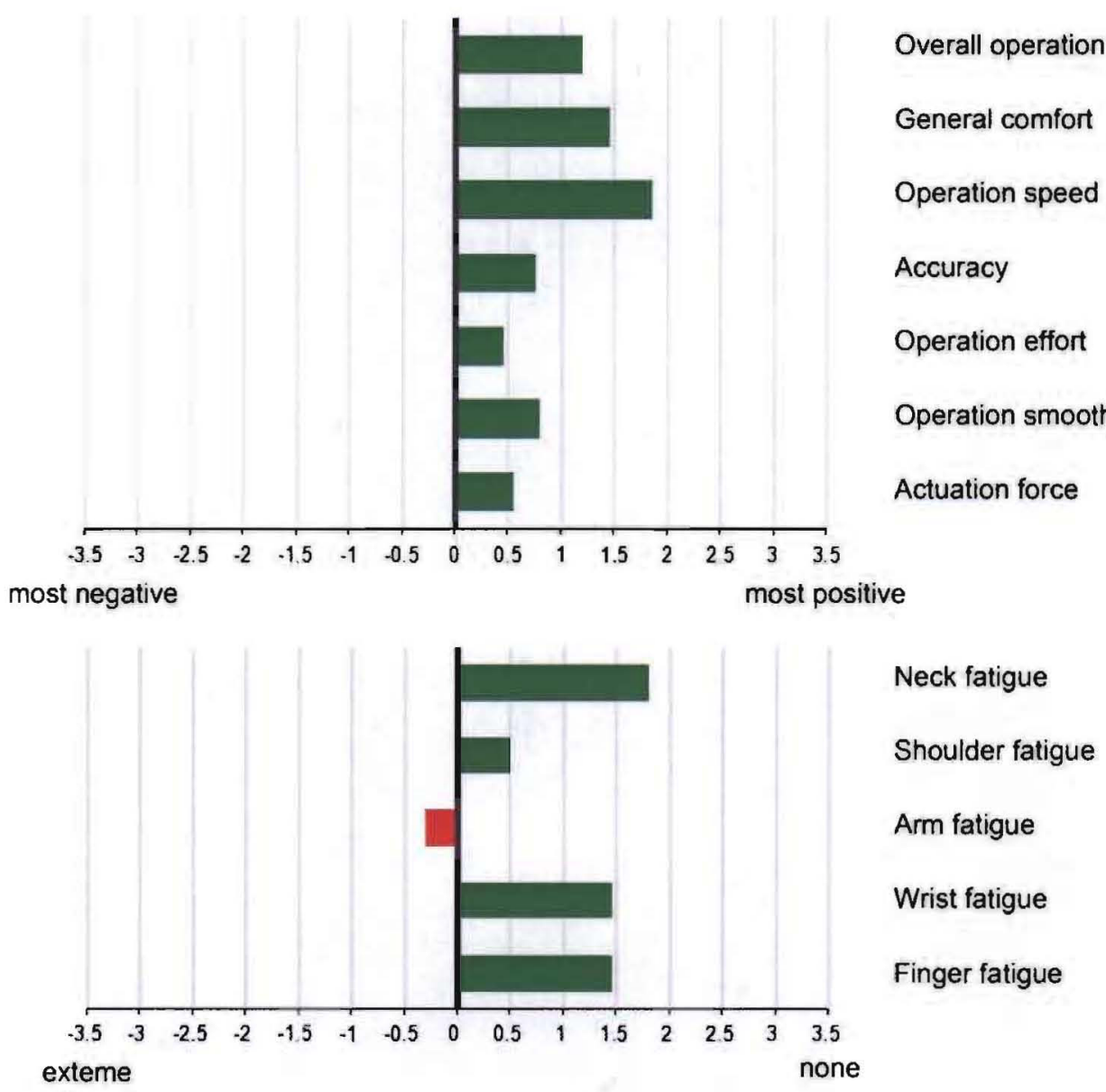

Fig. 9. Subjective user rating for non-tactile feedback.

Overall operation
General comfort
Accuracy
Operation speed
Operation smoothness
Operation effort
Actuation force
Finger fatigue
Wrist fatigue
Arm fatigue
Shoulder fatigue
Neck fatigue

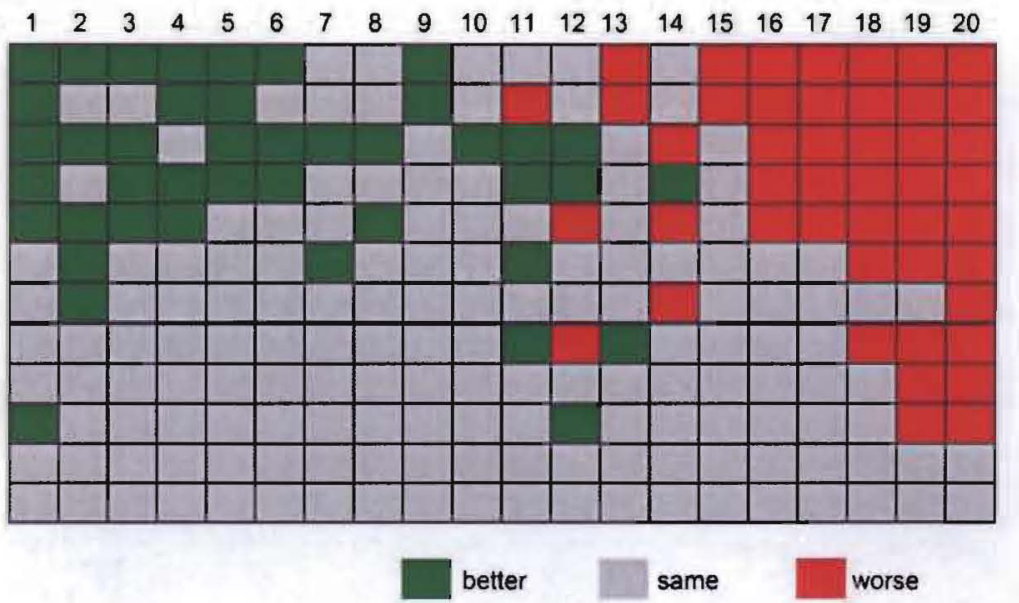

Fig. 10. Relative user rating for tactile feedback. 
performance since the additional information channel can complement or substitute visual information our results show no significant effect in terms of effective index of performance and even a small but significant higher error rate for horizontal target alignment when using tactile feedback. One explanation might be that participants did not take advantage of the additional feedback since they relied more on their visual observations when initiating a selection, as this is more common and known. So, the performance did not show a difference as tactile feedback might have simply be tolerated but not used by the participants. However in the negative case, the additional tactile feedback could even interfere with the visual information. We know from cognitive science that tactile and visual stimulations are not processed with the same lag and that velocity and measured reaction time differ [19]. Users may react irritated if the same information (target reached) gets delivered from different channels at different times. This effect may not only be due to the processing of the stimuli, but also to variations originating from technical reasons, e.g. refresh rate of the display, and the fact that visual feedback does not come unexpected to the participant. Moreover, some participants mentioned that they felt to be set under pressure by the additional feedback, what could also be a reason for the slight drawback considering the error rate. Basically, the findings of previous research on tactile feedback could not be directly transferred to hand gesture interaction. Our empirical results showed no benefit of tactile feedback at least in our test setting, in which visual and tactile information were provided to code the same event redundantly.

Furthermore, our study confirmed the findings of Dennerlein et al. [8] concerning the effect of movement direction on user performance. The results showed with $2.99 \mathrm{bits} / \mathrm{s}$ horizontal vs. $2.49 \mathrm{bits} / \mathrm{s}$ vertical a significant effect in terms of the effective index of performance. However, in contrast to their results, the tactile feedback did not compensate the differences between horizontal and vertical target alignment which could be due to the fact that in Dennerlein's study force feedback restricted mouse movement whereas tactile feedback in this evaluation only served as an additional information but did not physically hinder users in their movement and provided no additional guidance to improve the lower physical performance during vertical movements. Another reason for the differences in performance might be the combination of the gestures used. Performing the selection gesture could lead to a slight repositioning of the cursor, due to correlated movements at the back of the hand which are captured by the hand target used for gathering the orientation and position of the hand. When holding the inside of the hand facing the floor while interacting, this could affect the performance measure of trials for vertically arranged targets, but not for horizontally arranged targets. However this is less likely for being the main reason, as a lower performance for vertical movement directions could also be observed for participants holding the inside of the hand facing the left wall.

\section{Conclusion}

In analogy to human non-verbal gestural communication, we introduced hand gesture interaction as a natural and flexible interaction technique for large, high-resolution displays. Based on previous findings of Kendon [11] and the experimental results of Vogel and Balakrishnan [21], we identified suitable gestures for pointing and selection tasks and realized gesture recognition in combination with a commercial finger tracking device. Since relying solely on the visual information might be difficult in such a flexible setting, where users might move around in front of the large high-resolution display. we combined the hand gesture interaction with tactile feedback. The main contribution of this paper is the evaluation of the effect of proactive tactile feedback on the usability of hand gesture interaction. The findings from $[1,18]$ discussed in Section 2 suggest that such proactive tactile feedback may improve user performance since the additional information channel can complement or substitute visual information. However, our results show no significant effect in terms of effective index of performance and even a small but significant higher error rate for horizontal target alignment when using tactile feedback. Besides, the non-tactile version of our hand gesture interaction was very well received by the participants, with 11 positive and only one negative rated item on the ISO satisfaction questionnaire. Also, the effective index of performance with a mean of $2.53 \mathrm{bits} / \mathrm{s}$ for vertical and $3 \mathrm{bits} / \mathrm{s}$ for horizontal target alignment is promising and suggests that hand gesture interaction provides an adequate and valuable interaction technique for large, high-resolution displays. The significant influence of target alignment on user performance suggests that the effect of movement direction should be considered when designing user interfaces and interaction techniques for hand gesture interaction at large highresolution displays.

Regarding future research, we think that a more systematical understanding and analysis of tactile feedback is needed. While our classification in proactive and retroactive feedback based on the current literature is a first start, our results suggest that there are clearly additional factors that influence the utility. It might even be that the technical implementation of the tactile feedback plays an important role - while it is quite common for mobile phones to be equipped with some kind of vibration technique, it might be at first rather inconvenient to feel a vibration directly at the fingertips. We suggest the intensified use of longitudinal designs for future studies, which can help to further clarify the influence of such factors.

\section{Acknowledgements}

The current research was done in the context of the project inteHRDis which is part of the research association "Information at your fingertips - Interactive Visualization for Gigapixel Displays", supported by the program Information Technology Baden-Württemberg 
(BW-FIT). This work was also supported by the DFG Research Training. Group GK-1042 "Explorative Analysis and Visualization of Large Information Spaces". We thank all members of the project inteHRDis, especially Hans-Joachim Bieg for his valuable comments and suggestions.

\section{References}

[1] M. Akamatsu IS. MacKenzie Movement characteristics using mouse with tactile and force feedback, International Journal of Human-Computer Studies 45 (4) (1996) 483-493.

[2] R. Ball, C. North, D.A. Bowman, Move to improve: promoting physical navigation to increase user performance with large displays, in: $\mathrm{CHI}$ '07: Proceedings of the SIGCHI Conference on Human Factors in Computing Systems, ACM, New York, NY, USA 2007. pp. 191-200.

[3] R. Ball, M. Varghese, A. Sabri, E.D. Cox, C. Fierer, M. Peterson, B. Carstensen, C. North, Evaluating the benefits of tiled displays for navigating maps, in: IASTED-HCI05: Proceedings of the International Conference on Human-Computer Interaction, 2005 pp. $66-71$.

[4] J. Barrett, H. Kruger, Performance effects of reduced proprioceptive feedback on touch typists and casual users in a typing task, Behaviour and Information Technology 13 (6) (1994) 373-381.

[5] T. Baudel, M. Beaudouin-Lafon, Charade: remote control of objects using free-hand gestures, Communications of the ACM 36 (7) (1993) 28-35.

[6] R.A. Bolt, Put-that-there: voice and gesture at the graphics interface, in: SIGGRAPH '80: Proceedings of the Seventh Annual Conference on Computer Graphics and Interactive Techniques, ACM. New York. NY, USA, 1980, pp. 262-270

[7] S. Brewster, F. Chohan, L. Brown, Tactile feedback for mobile interactions, in: $\mathrm{CHI}$ 07: Proceedings of the SIGCHI Conference on Human Factors in Computing Systems, ACM, New York, NY, USA 2007, pp. 159-162.

[8] J.T. Dennerlein, D.B. Martin, C. Hasser, Force-feedback improves performance for steering and combined steering-targeting tasks, in: CHI '00: Proceedings of the SIGCHI Conference on Human Factors in Computing Systems, ACM, New York, NY, USA, 2000, pp. $423-429$

[9] C. Forlines, R. Balakrishnan, Evaluating tactile feedback and direct vs, indirect stylus input in pointing and crossing selection tasks, in: CHI '08: Proceeding of the Twenty-Sixth Annual SIGCHI Conference on Human Factors in Computing Systems, ACM, New York, NY, USA 2008, pp. 1563-1572.
[10] ISO 9241. Ergonomic requirements for office work with visual display terminals (VDTs) - Part 9: requirements for non-keyboard input devices, 2000.

[11] A. Kendon, Gesture: Visible Action as Utterance, Cambridge University Press, Cambridge, 2004.

[12] W.A. König, H.-J. Bieg. T. Schmidt, H. Reiterer, Position-independent interaction for large high-resolution displays, in: $\mathrm{IHCl}^{\circ} 07$ : Proceedings of the IADIS International Conference on Interfaces and Hurnan Computer Interaction 2007. IADIS Press, July 2007. pp. 117-125.

[13] W.A. König, J. Böttger, N. Völzow, H. Reiterer, Laserpointer Interaction between art and science, in: IUI08: Proceedings of the 13th International Conference on Intelligent User Interfaces, ACM Press, New York. NY. USA, January 2008, pp. 423-424.

[14] W.A. König, J. Gerken. S. Dierdorf, H. Reiterer, Adaptive pointing implicit gain adaptation for absolute pointing devices, in: $\mathrm{CHI} 09$ Extended Abstracts on Human Factors in Computing Systems, Work-In-Progress Session, ACM, New York, NY, USA, April 2009.

[15] M. McGuffin, R. Balakrishnan, Acquisition of expanding targets, in: CHI'O2: Proceedings of the SIGCHI Conference on Human Factors in Computing Systems: Changing our World, Clianging Ourselves. ACM, Minneapolis, MN, USA, April 2002, pp. 57-64.

[16] E. Mutschler, H.-G. Schaible, P. Vaupel, Anatomie-PhysiologiePathologie des Menschen, Wissenschaftliche Verlagsgesellschaft mbH Stuttgart, 2007.

[17] G. Robertson, M. Czerwinski, P. Baudisch, B. Meyers, D. Robbins, G. Smith, D. Tan, The large-display user experience, IEEE Computer Graphics and Applications 25 (4) (2005) 44-51.

[18] R. Scheibe, M. Moehring, B. Froehlich, Tactile feedback at the finger tips for improved direct interaction in immersive enviromments, in: 2007 IEEE Symposium on 3D User Interfaces. IEEE Computer Society Press, Silver Spring. MD, 2007, pp. 123-130.

[19] N, Serge, On the speed of different senses and nerve transmission by Hirsch (1862), Psychological Research 59 (4) (1997) 261-268.

[20] R.W. Soukoreff, I.S. MacKenzie, Towards a standard for pointing device evaluation, perspectives on 27 years of Fitts' Law research in $\mathrm{HCl}$, International Journal of Human-Computer Studies 61 (6) (2004) 751-789.

[21] D. Vogel, R. Balakrishnan, Distant freehand pointing and clicking on very large, high resolution displays, in: UIST '05: Proceedings of the 18th Annual ACM Symposium on User Interface Software and Technology, ACM, New York, NY, USA, 2005, pp. 33-42.

[22] B. Yost, Y. Haciahınetoglu. C. North, Beyond visual acuity: the perceptual scalability of information visualizations for large displays, in: $\mathrm{CHI}$ '07: Proceedings of the SIGCHI Conference on Human Factors in Computing Systems, ACM. New York, NY, USA, 2007. pp. 101-110. 